\title{
Real-time ultra-wideband video streaming in long-reach passive optical networks with wireless radiation in the 10 and $60 \mathrm{GHz}$ Bands
}

\author{
Tiago M. F. Alves ${ }^{1 *}$, Maria Morant ${ }^{2}$, Adolfo V. T. Cartaxo $^{1}$, Roberto Llorente ${ }^{2}$, \\ Pierre Cluzeaud $^{3}$, and Rakesh Sambaraju ${ }^{4}$ \\ ${ }^{1}$ Instituto de Telecomunicações, Department of Electrical and Computer Engineering, \\ Instituto Superior Técnico, Universidade de Lisboa, 1049-001 Lisbon, Portugal \\ ${ }^{2}$ Nanophotonics Technology Centre, Universitat Politècnica de València, 46022 Valencia, Spain \\ ${ }^{3}$ THALES Communications, Gennevilliers, France \\ ${ }^{4}$ Science and Technology Department, Corning Incorporated, One Riverfront Plaza, Corning, NY 14831, USA \\ ${ }^{*}$ Corresponding author: tiago.alves@lx.it.pt
}

Received May 29, 2013; accepted August 6, 2013; posted online September 29, 2013

\begin{abstract}
Real-time video streaming using ultra-wideband (UWB) technology is experimentally demonstrated along long-reach passive optical networks (LR-PONs) with different wired and wireless reaches. Experimental tests using external and direct modulation with UWB wireless radiation in the 10- and $60-\mathrm{GHz}$ bands are performed. An ultra-bendable fiber is also considered for a last-mile distribution. The video quality at the output of the optical fiber infrastructure of the LR-PON is assessed using the error vector magnitude $(\mathrm{EVM})$, and the link quality indicator (LQI) is used as a figure of merit after wireless radiation. An EVM below $-17 \mathrm{~dB}$ is achieved for both externally and directly modulated LR-PONs comprising up to $125 \mathrm{~km}$ of optical fiber. EVM improvement is observed for longer LR-PONs when directly modulated lasers (DMLs) are used because of the amplitude gain provided by the combined effect of dispersion and DML's chirp. Compared with optical back-to-back operation, the LQI level degrades to the maximum around $20 \%$ for LR-PONs ranging between 75 and $125 \mathrm{~km}$ of fiber reach and with a wireless coverage of $2 \mathrm{~m}$ in the 10-GHz UWB band. The same level of LQI degradation is observed using the 60-GHz UWB band with a LR-PON integrating $101 \mathrm{~km}$ of access network, a last-mile distribution using ultra-bendable fiber, and a 5.2-m wireless link.
\end{abstract}

OCIS codes: 060.0060, 060.2330.

doi: 10.3788/COL 201311.100605.

Ultra-wideband (UWB) technology has been proposed as an attractive radio solution for use in short-range wireless networks because of the advantages of UWB, such as high data-rate broadcasting, tolerance to multipath fading, possibility of coexistence with other technologies, and low power consumption allowing smallsize/low-cost integration ${ }^{[1]}$. Impulse-radio and orthogonal frequency division multiplexing (OFDM) have been proposed as UWB signal modulation formats ${ }^{[1]}$. However, the OFDM-UWB solution shows enhanced features for telecommunication purposes. These features include high flexibility for providing multiple access inherent to multi-band techniques and low band limitations in the UWB transceiver ascribed to the $528-\mathrm{MHz}$-wide channelization of OFDM-UWB signals.

The band used for UWB technology was initially allocated between 3.1 and $10.6 \mathrm{GHz}$ with a maximum equivalent isotropic radiated power (EIRP) of -41.3 $\mathrm{dBm} / \mathrm{MHz}^{[2-4]}$. Lately, the transmission of UWB signals in the unlicensed $60-\mathrm{GHz}$ band has been appointed as a viable and preferable solution for use in wireless personal area networks (WPANs). This preference is supported by the wireless reach extension (enabled by the maximum allowed EIRP of $60-\mathrm{GHz}$ band) and by the improvement in data transfer speeds ${ }^{[5-7]}$.

Transmission of UWB signals through the fiber-to-thehome (FTTH) infrastructure has been proposed and suc- cessfully demonstrated as a powerful solution to increase the UWB coverage area ${ }^{[8]}$. The extension of FTTH reaches up to $100 \mathrm{~km}$ is emerging as a new target for some network operators particularly concerned about delivering high-bandwidth services to users' premises in sparse geographical areas and about decreasing the number of central offices in dense geographical areas ${ }^{[9]}$. Several works have demonstrated the transmission of UWB signals along these so-called long-reach passive optical networks (LR-PONs). In Ref. [10], the simultaneous distribution of three UWB bands along $60 \mathrm{~km}$ of singlemode fiber (SMF) is demonstrated with off-the-shelf devices. This fiber reach is further extended to $75 \mathrm{~km}$ in Ref. [11] by a phase compensation technique to overcome dispersion-induced power fading. In Ref. [11], an UWB wireless transmission distance of $1.5 \mathrm{~m}$ is used. Recently, UWB broadcasting along LR-PONs reaching $100 \mathrm{~km}$ has been reported through numerical simulation using fixed optical dispersion compensation or chirped electrooptic converters (EOCs) to mitigate power fading ${ }^{[12,13]}$. The distribution of UWB signals along 100-km-long wavelength-division multiplexing (WDM) LR-PONs has also been experimentally demonstrated ${ }^{[14,15]}$. Although UWB signals coexisting with other wireless and wired OFDM-based signals in LR-PONs with fiber coverage exceeding $60 \mathrm{~km}$ has been addressed in these works, wireless transmission within users' premises is not performed. 
The distribution of UWB radio signals in the $60-\mathrm{GHz}$ band has also been reported ${ }^{[7,16]}$. In Ref. [16], a fiber transmission distance of $50 \mathrm{~m}$ is demonstrated by direct transmission of $60-\mathrm{GHz}$ UWB signals in the fiber. The fiber reach significantly increases to $40 \mathrm{~km}$ in the work reported in Ref. [7]. The FTTH network is also fully integrated with an ultra-bendable in-building fiber distribution reaching $500 \mathrm{~m}$ and with a wireless network comprising $5 \mathrm{~m}$ between antennas.

In this letter, we experimentally demonstrate the UWB real-time video streaming to user premises served by a LR-PON reaching $125 \mathrm{~km}$ of optical fiber and $5.2 \mathrm{~m}$ of wireless radiation. Demonstration is performed for externally and directly modulated LR-PONs, as well as for different wired and wireless coverage distances. UWB wireless radiation in the $10-$ and $60-\mathrm{GHz}$ bands is also considered.

Figure 1(a) shows a schematic of the hybrid wiredwireless LR-PON used to provide the video service to end users. This LR-PON is a simplified version of the WDM LR-PON proposed in Ref. [15] where different ONUs are served by different wavelengths. These different wavelengths ensure that different contents may be delivered to different users without significant requirements of electrical bandwidth at the optical network unit (ONU) side.

At the optical line termination (OLT), the video running in a laptop is sent via ethernet connection to the transmitter board of a Wisair development kit (DVK) to generate the UWB signal. The configuration parameters of UWB generation are controlled by the WisMan software. With this software, the UWB bands used for video streaming are adequately selected by time-frequency coding (TFC). In addition, the bit-rate and power of the UWB signal at the output of the UWB transmitter can be selected. The bit-rate of the Wisair DVK is bounded by 53.3 and $200 \mathrm{Mb} / \mathrm{s}$. The OLT also comprises a multiOFDM signal generator. This generator is implemented in Matlab and used to create different wired and wireless OFDM-based signals commonly assigned to access networks. The block diagram of each OFDM signal generator is depicted in Fig. 1(b). The bundle of OFDM-based signals is electrically generated using an arbitrary waveform generator (AWG) and enables performance assessment of the UWB video streaming when coexisting with other signals along the LR-PON. The transmission of the UWB video service alone or coexisting with other signals is controlled by switch S1 (Fig. 1(a)). Prior to electricalto-optical conversion, the signal is amplified and filtered to reduce the noise power using an amplifier with a gain of $26 \mathrm{~dB}$ and a low-pass filter (LPF) with $-3-\mathrm{dB}$ bandwidth of $8.2 \mathrm{GHz}$. External and direct modulation are the electrical-to-optical conversion approaches tested in this work. External modulation is accomplished using a distributed feedback laser (DFB) and a chirpless singlearm 10-Gb/s Mach-Zehnder modulator (MZM) biased at the quadrature. The directly modulated laser (DML) is a multi-quantum well DFB laser characterized by a threshold current $I_{\mathrm{th}}=8.1 \mathrm{~mA}$, a bias current $I_{\mathrm{b}}=30 \mathrm{~mA}$, a chirp parameter of 2.6, and an intensity modulation (IM) response bandwidth of about $4 \mathrm{GHz}^{[17]}$. Switch $\mathrm{S} 2$ is used to select between the MZM and DML.

The feeder fiber connecting the OLT to the remote node $(\mathrm{RN})$ comprises $75 \mathrm{~km}$ of $\mathrm{SMF}$. At the $\mathrm{RN}$, the optical signal is amplified and filtered to compensate for fiber loss and to reduce amplified spontaneous emission noise, respectively. The wavelength router is implemented through an optical filter with a -3 -dB bandwidth of $17 \mathrm{GHz}$. We notice that these experiments are developed without using any kind of optical dispersion compensation at the RN. This case is possible because only the UWB band group 1 is available in the Wisair DVK and its higher frequency is $4.75 \mathrm{GHz}$. Consequently, degradation caused by fiber dispersion-induced power fading is reduced ${ }^{[18]}$. If other UWB bands with higher central frequencies are also delivered to user premises, system performance is remarkably impaired by power fading. In this case, optical dispersion compensation or single-sideband transmission must be used.

The distribution network connects the RN to the ONU. The average optical power at the input of the feeder and distribution fibers is adjusted to levels below $0 \mathrm{dBm}$ to ensure negligible degradation due to fiber nonlinearity. Distribution fiber lengths ranging between 0 and $50 \mathrm{~km}$ are analyzed to emulate different LR-PON coverage scenarios. Additionally, some tests are performed considering signal transmission along a last-mile fiber distribution $^{[19]}$. In this case, the distribution network is composed of the distribution fiber and the last-mile distribution fiber interconnected by the adapter, as shown in Fig. 1(a). This last-mile distribution fiber is used to deliver services to end users and represents deployment constraints associated with unavoidable fiber bends realized to install fibers up to user premises. These deployment constraints are emulated by rolling a ClearCurve ${ }^{\circledR}$ fiber around a board marker with 20 bends and a bend radius of $7.5 \mathrm{~mm}$, as shown in Fig. 1(c). The reach of the ultra-bendable fiber is $1 \mathrm{~km}$. The last-mile distribution fiber is inserted into the LR-PON under analysis through switch S3.

At the ONU, the variable optical attenuator (VOA) is used to set the average optical power of the signal arriving at the photodetector to $-11 \mathrm{dBm}$. Photodetection is performed using a $10-\mathrm{GHz}$ PIN with a responsivity of $0.75 \mathrm{~A} / \mathrm{W}$ and a transimpedance amplifier stage. The received current is further amplified (gain of $21 \mathrm{~dB}$ ) and filtered by a LPF with a $-3 \mathrm{~dB}$ bandwidth of $8.2 \mathrm{GHz}$. After filtering, a variable electrical attenuator (VEA) is used to adjust the EIRP of the signal to be radiated to meet the UWB power mask constraints. At this point, one of the following four situations can occur depending on the desired analysis. 1) The resulting signal is digitized by an Agilent real time oscilloscope operating at $10 \mathrm{Gs} / \mathrm{s}$ and the error vector magnitude (EVM) of the received video after transmission along the optical part of the hybrid wired-wireless LR-PON is evaluated with Agilent 89600 vector signal analyzer software. If the other OFDM-based signals are transmitted in coexistence with the UWB video signal, they are processed offline using Matlab for demodulation, and the EVM of each OFDM-based interfering signal is calculated. 2) The resulting signal is directly connected to the receiver device of the Wisair DVK where the video stream is recovered and displayed on a TV screen. In this case, the quality of the received signal is assessed by WisMan software. Video quality is quantified using the link quality indicator (LQI), which represents the quality of received 
packets. The received signal strength indicator (RSSI), that measures the power present in the received signal, is also provided by the software. 3) The resulting signal is applied to an UWB antenna, radiated to emulate the wireless transmission inside the user premises, captured by another antenna, and delivered to the receiver board of Wisair DVK for video stream recovery. Subsequently, the signal is displayed on TV, and video quality is assessed using the RSSI and LQI as figures of merit. 4) This situation is similar to the one described in 3 ). The main difference is that the UWB signal is radiated in the $60-\mathrm{GHz}$ band rather than in the $10 \mathrm{GHz}$ band. This case is enabled using a 10 to $60 \mathrm{GHz}$ upconverter before the transmitter antenna and a 60 to $10 \mathrm{GHz}$ downconverter after the receiver antenna, as shown in Fig. 1(a). The desired operation case is selected using switch S4 (Fig. $1(\mathrm{a}))$.

The effect of wired LR-PON coverage on video performance is assessed to examine the degradation induced by the joint effect of EOC-induced distortion and fiber dispersion. In addition, the interference caused by the transmission of UWB signals coexisting in the LR-PON with other OFDM signals is discussed.

UWB standard establishes $-14.5 \mathrm{~dB}$ as the EVM limit for UWB signals ${ }^{[4]}$. This EVM limit is defined at the output of the wireless transmitter antenna to ensure that the signal quality is sufficient to establish the wireless link. Thus, in this work, we use this limit as a quality reference for the maximum acceptable EVM at the output of the optical fiber infrastructure.

Studies considering the two EOCs (MZM and DML) are performed, no wireless link is established during the experiments, and the last-mile fiber distribution is not used. The analysis is firstly performed considering the transmission of the UWB signal alone along the LR-PON, i.e., $\mathrm{S} 1$ is opened.

Figure 2(a) shows the power spectral density (PSD) of the UWB signal used to transmit the video service at

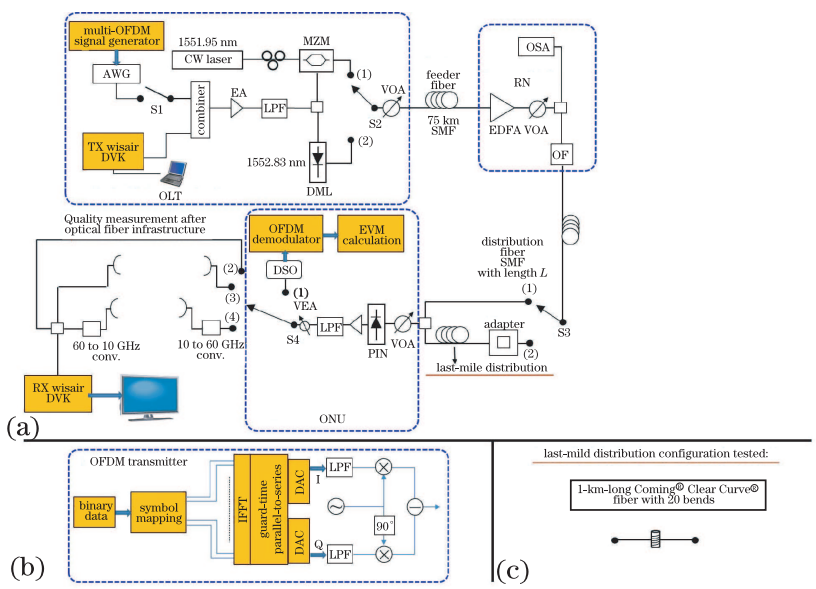

Fig. 1. (a) Schematic of the hybrid wired-wireless LR-PON used to provide the video service to end users. (b) Block diagram of the OFDM transmitter. (c) Last mile distribution configuration tested in the experiments. CW: continuous wave; DSO: digital storage oscilloscope; EA: electrical amplifier; EDFA: erbium doped fiber amplifier; OF: optical filter; OSA: optical spectrum analyzer; RX: receiver; TX: transmitter.
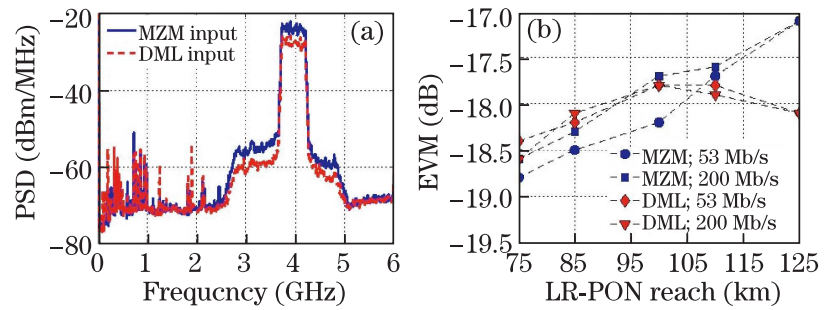

Fig. 2. (a) PSD of the UWB signal applied to the MZM and DML. (b) EVM of the UWB signal prior to wireless radiation when external and direct modulation is used at the OLT side as an electro-optic conversion approach.

the EOC input. Spectra were obtained considering the power parameter of WisMan software set to 0 and -5 $\mathrm{dBm}$, respectively, when the MZM or DML was used. These power levels correspond to the optimized system operation for each EOC case. The UWB signals presented in Fig. 2(a) correspond to UWB band \#2, centered in $3.96 \mathrm{GHz}$, as the TFC 6 is selected in WisMan software.

Figure 2(b) depicts the EVM of the received UWB signal as a function of the total LR-PON reach considering external and direct modulation. Results for 53 and 200 $\mathrm{Mb} / \mathrm{s}$ are presented. Figure 2(b) shows that EVM levels below $-17 \mathrm{~dB}$ are obtained regardless of the reach of the LR-PON, the bit-rate of the UWB signal, and the EOC used. These EVM levels still comprise a good margin to the EVM threshold of the ECMA standard $(-14.5 \mathrm{~dB})$.

Figure 2(b) also shows that, for the external modulation case and when compared with the reach of $75 \mathrm{~km}$, an EVM degradation reaching almost $2 \mathrm{~dB}$ occurs when the reach of the LR-PON is $125 \mathrm{~km}$. The reason for such degradation is easily understood by inspecting the received UWB signal spectra shown in Fig. 3(a). The amplitude of subcarriers of the UWB signal with higher frequency is reduced. This reduction increases with increased subcarrier frequency and also when longer LRPONs are considered. These effects are commonly observed when degradation is due to power fading induced by fiber chromatic dispersion ${ }^{[18]}$.

Figure 2(b) shows that when the DML is used as EOC, an EVM fluctuation not exceeding $0.7 \mathrm{~dB}$ is observed when the reach of the LR-PON increases from 75 to $100 \mathrm{~km}$. Contrary to the MZM case, a slight EVM improvement not exceeding $0.3 \mathrm{~dB}$ is also noticed when the reach of the LR-PON increases from 100 to 125 $\mathrm{km}$. This behavior is attributed to the joint effect of distortion and chirp introduced by the DML, combined with chromatic dispersion of the link. This combined effect leads to an IM response that is translated to signal frequency-dependent amplitude gain at the ONU side after photodetection ${ }^{[17]}$, as shown in the spectra presented in Fig. 3(b).

Subsequently, the effect of transmitting the UWB video service through the LR-PON in coexistence with other OFDM-based potentially interfering signals recently proposed for next-generation access networks ${ }^{[14]}$ is assessed. The reach of the LR-PON is $100 \mathrm{~km}$, and the received signal is sent to the scope. The interference study is performed considering the MZM. This option is supported by the results shown in Fig. 2(b) for a 100km-long LR-PON, i.e., despite the fact that the EVM 


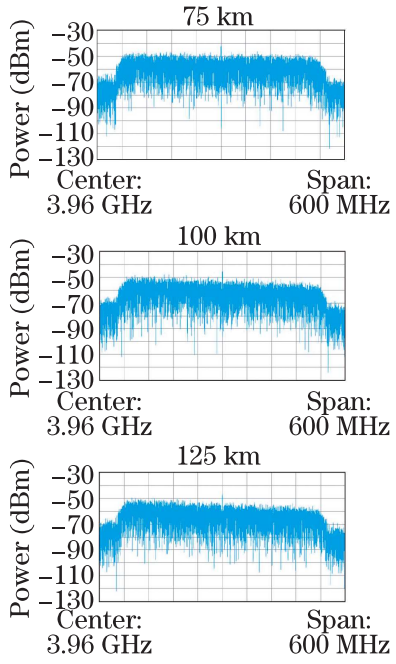

(a)
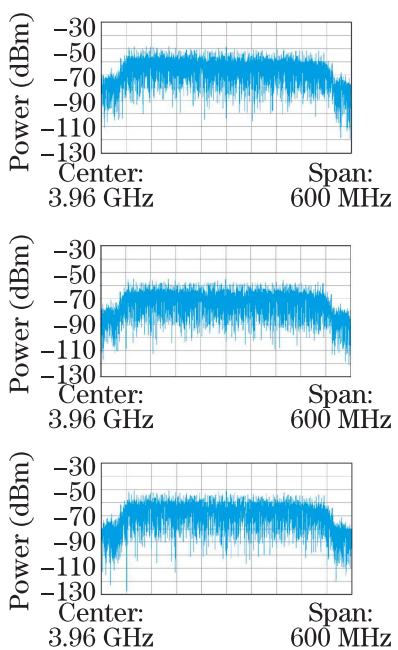

(b)
Fig. 3. Spectra of the received UWB signal for three distribution fiber reaches and considering (a) external and (b) direct modulations. The UWB signal bit-rate is $200 \mathrm{Mb} / \mathrm{s}$.

of the $200-\mathrm{Mb} / \mathrm{s}$ UWB signal is similar when the MZM or the DML is used, the MZM allows obtaining a better EVM for the $53-\mathrm{Mb} / \mathrm{s}$ bit-rate option.

Two wireless and one wired OFDM signals are the interfering signals. The wireless signals are based on the long term evolution (LTE) and wireless interoperability for microwave access (WiMAX) technologies, whereas the wired signal is a custom OFDM-based signal that provides a capacity similar to that of standard Gigabit Ethernet (GbE). The main features of these signals are presented in Ref. [15]. The EVM limits defined in the corresponding wireless standards are: $-15.1 \mathrm{~dB}$ for LTE and $-20 \mathrm{~dB}$ for WiMAX. In the case of the OFDM-GbE, we select an EVM limit of $-11 \mathrm{~dB}$ corresponding to a bit error ratio of $10^{-4}$ in a noise-impaired quadrature phaseshift keying system. The PSD of the bundle of OFDM signals at the OLT side and at the ONU side are shown in Figs. 4(a) and (b) as a reference. The electrical power percentage distribution between the different OFDMbased signals shown in Fig. $4(\mathrm{a})$ is $19 \%$ for OFDMGbE signal, $5 \%$ for LTE signal, $10 \%$ for WiMAX signal, and $66 \%$ for UWB signal. These power percentages are chosen from a detailed numerical simulation study in which a set of optimum distributions that maximizes the difference between the EVM of each signal and the EVM of corresponding standards is identified. The power decay of signals with high frequency (UWB signal) noticeable in Fig. 4(b) is due to power fading and the limited frequency response of the system.

Figure 5 shows the constellations and corresponding EVMs of the received OFDM-GbE, LTE, WiMAX, and UWB signals at the output of the receiver LPF. Comparison of the EVMs of the UWB signal presented in Fig. 5 with the EVM obtained when the UWB signal is transmitted alone in the LR-PON (Fig. 2(b), MZM case) reveals a reduced EVM degradation not exceeding $1 \mathrm{~dB}$ of the UWB signal due to coexistence with other OFDM-based signals. Comparison between the EVMs of Fig. 5 and the EVM limits of each signal standard reveals also that the EVM of all signals of the OFDM bundle meets the EVM requirements stated in each standard.
This conclusion obtained with real-time video streaming in UWB signal agrees with the conclusions drawn in Refs. [14,15], where transmission using random binary sequences with off-line processing is performed.

The quality of the received video is subsequently assessed using the LQI as a figure of merit. Some results of the RSSI parameter are also presented. The effect of fiber and wireless reach on video quality is assessed. Video transmission along the optical fiber infrastructure in the $10-\mathrm{GHz}$ UWB band and the two types of EOCs are considered. In the case of the wireless reach, radiation in the 10 - and $60-\mathrm{GHz}$ bands is tested. The $60-\mathrm{GHz} \mathrm{UWB}$ radiation is achieved using the $10-60 \mathrm{GHz}$ transceivers at the output of the optical fiber infrastructure, as depicted in Fig. 1(a). The quality of the received video is also assessed considering the ultra-bendable ClearCurve ${ }^{\circledR}$ fiber to provide last-mile distribution.

In the following studies, the wireless transmission of the UWB signal is performed in the $10-\mathrm{GHz}$ band. Smallfootprint omni-directional Omron WXA-N2SL wideband monopole antennas with a gain of typically $2 \mathrm{dBi}$ are used. Experiments are accomplished for a 2-m distance between antennas because this is the maximum reach achieved with these antennas that still lead to acceptable visual quality of video streaming on the TVscreen. TFC 6 (UWB band \#2) and TFC 5 (UWB band \#1) are selected in WisMan software when the MZM or the DML are used as EOCs, respectively. TFC 6 is not used in both cases because, for the DML case, it leads to some pixelation effects on the video displayed on the TV-screen.

Table 1 summarizes the RSSI and LQI levels provided by WisMan software in back-to-back (b2b) operation (the
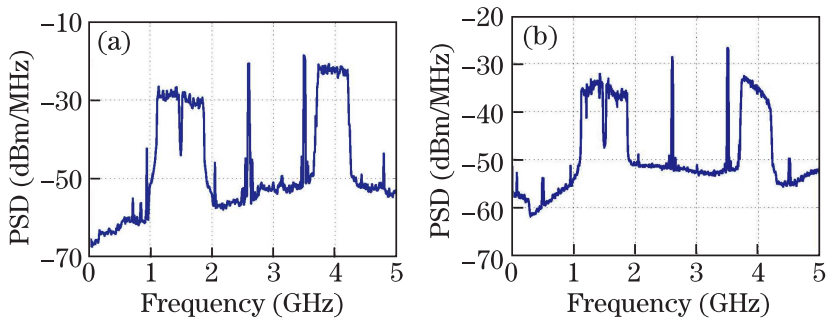

Fig. 4. Spectrum of the UWB signal in coexistence with OFDM-GbE, LTE, and WiMAX signals (a) at the input of the MZM and (b) after the PIN.

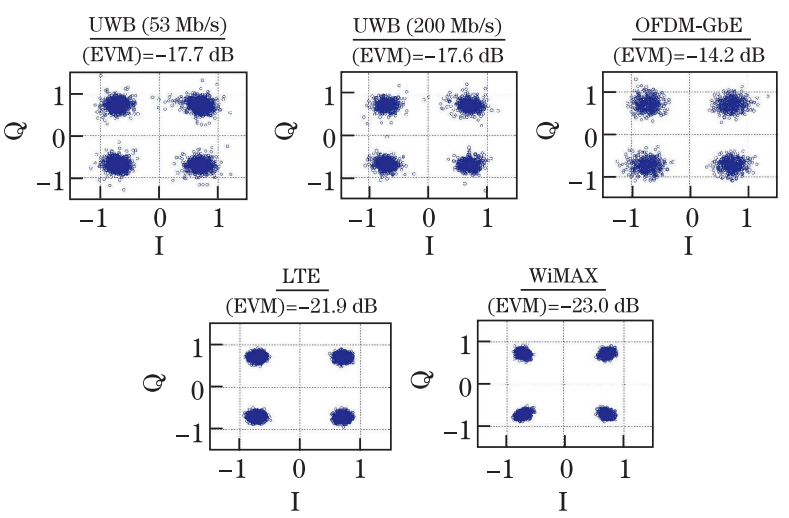

Fig. 5. Constellations of the UWB, OFDM-GbE, LTE, and WiMAX signals. 
Table 1. RSSI and LQI of the Video in b2b Operation. $(x ; y)$ Represents the Values Obtained for Bit-rate of 53 and $200 \mathrm{Mb} / \mathrm{s}$

\begin{tabular}{ccc}
\hline & MZM & DML \\
\hline RSSI & $(13 ; 15)$ & $(23 ; 23)$ \\
LQI & $(18 ; 19)$ & $(24 ; 24)$ \\
\hline
\end{tabular}

optical fiber is removed from the setup shown in Fig. 1(a) and without wireless transmission) for bit-rates of 53 and $200 \mathrm{Mb} / \mathrm{s}$ and considering the MZM or the DML as EOC. These results are used in the following sections as the reference levels for comparison. Although slight bit-rate dependence is observed in the performance results of Table 1 for the MZM case, this dependence is reduced and is attributed to slight deviations in the bias point of the MZM induced by temperature fluctuations in the laboratory. In the case of the DML, this effect is not observed because the DML incorporates a temperature controller.

Figure 6 shows results similar to those in Fig. 2(b) but using the LQI as video quality indicator rather than the EVM and considering the wireless transmission at user premises. The LQI values obtained in b2b operation with 53 and $200 \mathrm{Mb} / \mathrm{s}$ are also shown in Fig. 6 for the MZM and DML cases. Four main conclusions can be drawn from Fig. 6. (i) When the MZM is used, the video quality slightly degrades (LQI gets worse) when the LRPON reach increases because of the chromatic dispersioninduced power fading. (ii) In the case of using DMLs, the video quality may improve for longer LR-PONs because of the combined effect of DML's chirp, distortion, and chromatic dispersion. (iii) Although the video quality obtained for LR-PONs ranging between 75 and $125 \mathrm{~km}$ is worse than in b2b operation, the performance improves for longer LR-PONs when DMLs are used. (iv) A maximum LQI degradation of around 20\% compared with b2b operation is observed regardless of the EOC used and the LR-PON reach considered.

The $60-\mathrm{GHz}$ UWB wireless transmission is analyzed as follows. The $60 \mathrm{GHz}$ transceivers comprise a local oscillator, mixer, up/down converters, and antennas. The antennas are from TES Germany and have a gain of $16-18 \mathrm{dBi}$. The effective gain of the $60-\mathrm{GHz}$ transceiver is 22 and $20 \mathrm{~dB}$ in the transmitter and receiver branches, respectively. The transmitter board of the $60-\mathrm{GHz}$ transceiver provides UWB signals with an EIRP level $30 \mathrm{~dB}$ below the maximum EIRP allowed in the $60-\mathrm{GHz}$ band when the input signal exhibits the maximum EIRP permitted in the $10 \mathrm{GHz}$ band. The results presented in this section consider that the MZM is used as EOC to enable the selection of TFC 6 on WisMan software without having the pixelation effect on the TVscreen.

Table 2 presents the RSSI and LQI of the received video for three different LR-PON reaches and considering a 2$\mathrm{m}$ distance between antennas. Comparison between the results of Table 2 with the RSSI $(13 ; 15)$ and LQI $(18 ; 19)$ levels obtained in b2b operation indicates the following: (i) the RSSI is much higher after transmission along the LR-PON and the $2-\mathrm{m}$ radiation in the $60-\mathrm{GHz}$ band than in $\mathrm{b} 2 \mathrm{~b}$ operation because of the signal power increase provided by the $60-\mathrm{GHz}$ UWB transceivers; (ii) the LQI of the received video after transmission along the LR$\mathrm{PON}$ is very similar to the one obtained in b2b operation. Therefore, no significant additional packet loss occurs because of optical fiber infrastructure impairments.

Figure 7 shows the video quality parameters as a function of the distance between $60-\mathrm{GHz}$ antennas and considering the bit-rate of the UWB signal set to $53 \mathrm{Mb} / \mathrm{s}$. These results are obtained considering a distribution fiber comprising $25 \mathrm{~km}$ and a last-mile ClearCurve ${ }^{\circledR}$ distribution fiber with a reach of $1 \mathrm{~km}$ and 20 bends, as depicted in Fig. 1(c). Therefore, the total reach of the LRPON is $101 \mathrm{~km}$. Additionally, the VOA at the PIN input is removed. Consequently, the average optical power arriving at the $\mathrm{PIN}$ is around $-8 \mathrm{dBm}$.

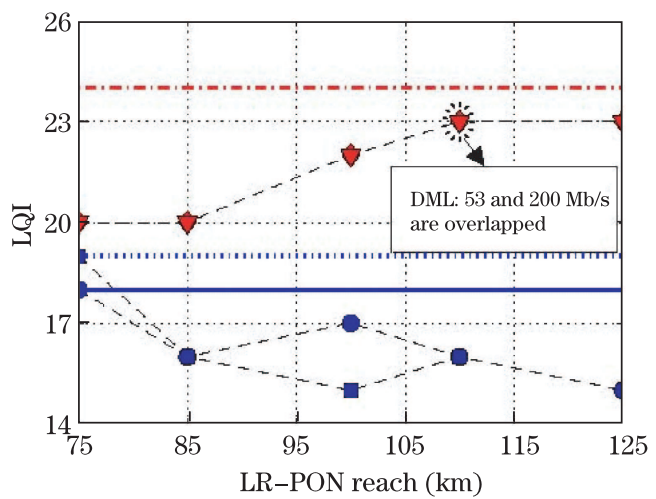

Fig. 6. LQI as a function of the reach of the LR-PON. Results obtained for the MZM and $53 \mathrm{Mb} / \mathrm{s}$ (circles), the MZM and $200 \mathrm{Mb} / \mathrm{s}$ (squares), the DML and $53 \mathrm{Mb} / \mathrm{s}$ (diamonds), and the DML and $200 \mathrm{Mb} / \mathrm{s}$ (triangles). Reference results obtained in b2b operation and with: the MZM and $53 \mathrm{Mb} / \mathrm{s}$ (continuous line), the MZM and $200 \mathrm{Mb} / \mathrm{s}$ (dotted line), the DML and 53 and $200 \mathrm{Mb} / \mathrm{s}$ (dashed-dotted line).

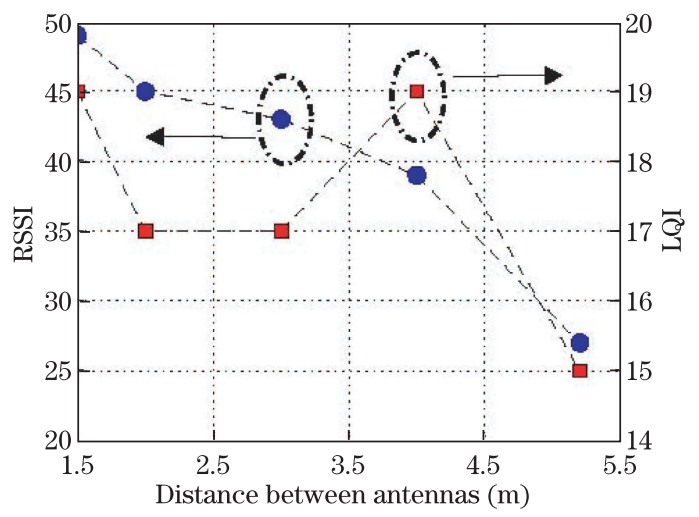

Fig. 7. RSSI and LQI of the received video as a function of the distance between $60-\mathrm{GHz}$ antennas. The total LR-PON reach is $101 \mathrm{~km}$, and the bit-rate is $53 \mathrm{Mb} / \mathrm{s}$.

Table 2. RSSI and LQI of the Video for Different

LR-PON Reaches with a 2-m Distance between Antennas. $(x ; y)$ Represents the values Obtained for Bit-rates of 53 and $200 \mathrm{Mbt} / \mathrm{s}$

\begin{tabular}{cccc}
\hline & $75 \mathrm{~km}$ & $100 \mathrm{~km}$ & $125 \mathrm{~km}$ \\
\hline RSSI & $(39 ; 39)$ & $(37 ; 37)$ & $(37 ; 37)$ \\
LQI & $(19 ; 17)$ & $(17 ; 17)$ & $(18 ; 17)$ \\
\hline
\end{tabular}


Figure 7 shows that the RSSI of the received video decreases with increased distance between antennas. This finding is mainly due to the power loss associated with free space propagation. Comparison between the results of Table 2 and Fig. 7 shows that different RSSI levels are achieved when the distance between antennas is $2 \mathrm{~m}$. This discrepancy is attributed to the different average optical power levels at the PIN input considered in the two situations. Figure 7 shows also that the LQI remains between 19 and 15 when the distance between antennas increases from 1.5 to $5.2 \mathrm{~m}$. These results indicate that the LQI degrades only from 18 (Fig. 6, in b2b operation) to 15 even considering UWB transmission through a LRPON reaching $101 \mathrm{~km}$ and radiation in a wireless link of $5.2 \mathrm{~m}$.

As a final remark, we emphasize that all experimental results presented in this work leads to video streaming on the TV-screen without any type of pixelation and with good visual quality.

In conclusion, experimental results addressing realtime video streaming using UWB technology along hybrid wired-wireless LR-PONs are demonstrated. LRPONs with coverage varying between 75 and $125 \mathrm{~km}$, electro-optic conversion performed with a conventional MZM or a low-cost DML, and considering UWB wireless radiation at user premises performed in the 10 - or 60 $\mathrm{GHz}$ bands are assessed and discussed. The dependence of the quality of received video service on the transmission of UWB signals in coexistence with other OFDMbased signals along the optical fiber infrastructure is also assessed. An EVM margin of the UWB signal relative to the limit of UWB standards exceeding $2.5 \mathrm{~dB}$ is demonstrated for LR-PONs reaching $125 \mathrm{~km}$ and using either MZMs or DMLs. EVM degradation of the received UWB signal not exceeding $1 \mathrm{~dB}$ is observed when the OFDMGbE, LTE, and WiMAX signals are transmitted in coexistence with the UWB signal along the LR-PON. Moreover, when the MZM is used, the LQI parameter degrades for longer LR-PONs because of the chromatic dispersioninduced power fading. Nevertheless, a LQI level of 15 is still achieved after a 125-km-long LR-PON and $2 \mathrm{~m}$ of wireless radiation in the $10 \mathrm{GHz} \mathrm{UWB}$ band. In the case of the DMLs, the LQI parameter can improve when the reach of the LR-PON increases because of the combined effect of DML's chirp, distortion, and chromatic dispersion. These results suggest that the DMLs may provide particular advantages to network operators for the coverage of specific users located at longer distances from the OLT with UWB technology. Finally, investigation of UWB radiation in the $60-\mathrm{GHz}$ band shows that a LQI of the video of 15 is also achieved after a 101-km-long LRPON using an ultra-bendable fiber for last-mile distribution and after radiation at user premises with antennas spaced by $5.2 \mathrm{~m}$.

This work was supported by the Fundação para a Ciência e a Tecnologia from Portugal under projects PEst-OE/EEI/LA0008/2013 and TURBO-
PTDC/EEATEL/ 104358/2008 and also by the European FIVER-FP7-ICT-2009-4-249142 project.

\section{References}

1. W. Siriwongpairat and K. Liu, Ultra Wideband Communications Systems-Multiband OFDM Approach(Wiley, Hoboken, 2008).

2. Federal Communications Comission, "Revision of part 15 of the comission's rules regarding ultra-wideband transmission systems: first report and order", (2002).

3. "Commission decision of 21 February 2007 on allowing the use of the radio spectrum for equipment using ultrawideband technology in a harmonised manner in the Community", Official Journal of the European Union (2007).

4. High Rate UltraWideband PHY and MAC Standard, 2nd ed. Geneve, Switzerland: ECMA Int., 2007.

5. Part 15.3: Wireless medium access control (MAC) and physical layer (PHY) specifications for high data rate wireless personal area networks, New York, USA: IEEE Computer Society, 2009.

6. High Rate $60 \mathrm{GHz}$ PHY, MAC and PALs, 2nd ed. Geneve, Switzerland: ECMA Int., 2010.

7. M. Beltrán, J. Jensen, X. Yu, R. Llorente, R. Rodes, M. Ortsiefer, C. Neumeyr, and I. Monroy, J. Sel. Areas Commun. 29, 1295 (2011)

8. M. Thakur, T. Quinlan, S. Dudley, M. Toycan, C. Bock, S. Walker, D. Smith, A. Borghesani, D. Moodie, R. Llorente, M. Ran, and Y. Ezra, in Proceedings of European Conference on Optical Communication Tu.4.F.4 (2008).

9. R. Davey, D. Grossman, M. Wiech, D. Payne, D. Nesset, A. Kelly, A. Rafael, S. Appathurai, and S. Yang, J. Lightwave Technol. 27, 273 (2009).

10. R. Llorente, T. Alves, M. Morant, M. Beltran, J. Perez, A. Cartaxo, and J. Marti, Photon. Technol. Lett. 20, 945 (2008).

11. W. Lin and Y. Hsu, in Proceedings of International Conference on Transparent Optical Networks Mo.C1.5 (2011).

12. T. Alves, M. Morant, A. Cartaxo, and R. Llorente, J. Sel. Areas Commun. 29, 1311 (2011).

13. T. Alves and A. Cartaxo, J. Lightwave Technol. 29, 2467 (2011).

14. M. Morant, T. Alves, A. Cartaxo, and R. Llorente, in Proceedings of Optical Fiber Communication Conference OW3B.2 (2012).

15. T. Alves, M. Morant, A. Cartaxo, and R. Llorente, Opt. Express 20, 13748 (2012).

16. B. Charbonnier, F. Lecoche, M. Weib, A. Stöhr, F. Dijk, A. Enard, F. Blache, M. Goix, F. Mallecot, D. Moodie, A. Borghesani, and C. Fors, in Proceedings of Optical Fiber Communication Conference OThO3 (2010).

17. J. Morgado, D. Fonseca, and A. Cartaxo, Opt. Express 19, 23601 (2011).

18. T. Alves and A. Cartaxo, Photon. Technol. Lett. 21, 158 (2009).

19. T. Alves, R. Sambaraju, A. Cartaxo, and A. Ng'oma, Chin. Opt. Lett. 11, 030606 (2013). 